Journal of Computer Science 6 (10): 1177-1184, 2010

ISSN 1549-3636

(C) 2010 Science Publications

\title{
Modeling A Multi-Compartments Biological System with Membrane Computing
}

\author{
${ }^{1}$ Ravie Chandren Muniyandi and ${ }^{2}$ Abdullah Mohd. Zin \\ ${ }^{1}$ School of Computer Science, \\ ${ }^{2}$ School of Information Technology, \\ Faculty of Information Science and Technology, \\ University Kebangsaan Malaysia, 43600 Bangi, Selangor, Malaysia
}

\begin{abstract}
Problem statement: Most of the biological systems have been hierarchical in structure with processes interacting between different compartments. Membrane computing formalism has provided modeling capabilities in representing the structure of biological systems. Approach: This study was carried to investigate the modeling of a multi-compartment biological system by using membrane computing formalism. The hormone-induced calcium oscillations in liver cells which was modeled with ordinary differential equation was used as a case study. The membrane computing model of this case study was verified and validated by using simulation strategy of Gillespie algorithm and the method of model checking using probabilistic symbolic model checker. The results of membrane computing model were compared to the ordinary differential equation model. Results: The simulation and model checking of membrane computing model of the biological case study showed that the properties of the multi-compartments biological system could be preserved with the membrane computing model. Membrane computing model could also accommodate the structure and processes of the multi-compartments biological system which were absent in the ordinary differential equation model. Conclusion: Membrane computing model provides a better approach in representing a multicompartment system and able to sustain the basic properties of the system. However appropriate value of parameters to represent the rules of the processes of the membrane computing model to manage the stochastic behavior should be formulated to meet the performance of the biological system.
\end{abstract}

Key words: Membrane computing, biological system, Gillespie algorithm, model checking

\section{INTRODUCTION}

Most of the biological systems are compartmentalized with each compartment provide different local environments that accommodate specific metabolic processes. The processes either occur within a compartment or by forming communication pathways to interact with one compartment to another via complex networks. The biological description of membrane computing formalism has been utilized to characterize and preserve the elements in biological systems. Membrane computing (Paun, 1998) is one of a modeling formalism for biological systems that provides a hierarchical structure for molecular computation in which membranes play an essential role for processes to pass in a regulated fashion within and across the membranes.

The research in this line shows that biological system can be modeled better using membrane computing than the conventional methods using mathematical representation such as Ordinary Differential Equation (ODE) (Bernardini and et al., 2006; Muniyandi and Abdullah, 2009). Simulation strategies based on membrane computing such as Gillespie Algorithm are also used to proof this claim (Muniyandi and Abdullah, 2010). Meanwhile, through model checking the operation of the model can be studied and consequently, the properties concerning the behavior of the biological system can be inferred as shown by Muniyandi et al. (2010).

In the multi-compartment biological system, the communication rules are used to execute the processes that interact between compartments. Each of these compartments could have similar or diverse activities to accomplish the collective behavior of the system. In some biological systems, the multi-compartments differ from one to another compartment by having their own chemical substances and processes to interact between

Corresponding Author: Ravie Chandren Muniyandi, School of Computer Science, Faculty of Information Science and Technology, University Kebangsaan Malaysia, 43600 Bangi, Selangor, Malaysia 
compartments. Therefore it is essential to formalize the compartmentalized biological system to describe the interacting processes and to represent the structure of the biological system in order to understand the behavior of the system in silico.

Population membrane computing introduced by Bernardini and Gheorghe (2004), was an attempt to represent multi-compartments where the links between the compartments are regulated by means of a specific set of communication rules. However this idea has to be explored in the real biological systems to determine the applicability of membrane computing formalism in representing multi-compartment biological system.

In this study, we investigate how a multicompartments system can be modeled by using membrane computing by taking a biological case study. The chosen case study is Hormone-induced Calcium Oscillations in Liver Cells which is a biological system with three different compartments. The membrane computing model of this case study is verified by using simulation and model checking techniques. The case study is simulated with simulation strategy of membrane computing of Gillespie Algorithm (Gillespie, 2001). The properties of the biological case study are validated by using the PRISM model checker (Kwiatkowska et al., 2002). Finally the results are discussed to determine the capability of membrane computing in modeling multicompartment biological system.

\section{MATERIALS AND METHODS}

Hormone-induced calcium oscillations in liver cells: Hormone-induced oscillations of the calcium concentration in the compartment of cytosol are used for frequency encoding of hormone signals. In liver cells, such calcium oscillations occur in response to stimulation by hormones, which cause an increase of the calcium concentration in cytosol. This biological system has three compartments: Extracellular, cytosol and Endoplasmic Reticulum (ER). Each of these compartments has their own task to meet the behavior of the system. The extracellular is either to flux in or out the calcium to or from the cytosol. Meanwhile, the ER moves calcium to and from cytosol. The overall system behavior will be determined by the level of calcium in cytosol. In order to facilitate these processes, there are five communication rules to interact between these compartments as follows: calcium flux from extracellular into the cytosol; calcium translocation from cytosol to ER; calcium translocation from ER to cytosol; calcium pumped out of cytosol into extracellular and hormone channeling from ER to cytosol.
This biological system is further elaborated by Somogyi and Stucki (1991). The system was modeled using ODE as $\frac{\mathrm{dx}}{\mathrm{dt}}=\mathrm{k}^{\prime} \mathrm{y}-\mathrm{kx}-\alpha \mathrm{f}[\mathrm{y}] \mathrm{x} \quad$ and $\frac{d y}{d t}=k x-k^{\prime} y+\alpha f[y] x+\gamma-\beta y$, with $f[y]=\frac{y^{n}}{a^{n}+y^{n}}$. and y are calcium concentration in ER and cytosol and calcium concentration in extracellular was assumed to be stable to enable consistent transportation of calcium into cytosol. $\mathrm{a}, \mathrm{k}^{\prime}, \mathrm{k}, \alpha, \gamma, \beta$ are parameters. $\mathrm{n}$ is an integer. Parameters were used to establish the weight to prioritize the execution of the processes. In the ODE model, the values of the constant parameters used were $\mathrm{k}^{\prime}=2, \mathrm{k}=0.01, \gamma=1, \beta=1, \mathrm{n}=4$ and the initial concentration for both $\mathrm{x}$ and $\mathrm{y}$ was 1 . Arbitrary values for parameters a and a were chosen to conclude whether the system was stable or unstable. A way to determine whether the system understimulated or overstimulated and a way to verify the low and high level of the hormone. The result in Somogyi and Stucki (1991) showed the different behavior of the system as illustrated in the Fig. 1: A and B show a stable sustained oscillations of $\mathrm{y}$ when the system was understimulated with low and high hormone. There were more spikes of oscillations with the experiment B when the hormone level increases; $\mathrm{C}$ and $\mathrm{D}$ show a stable nonoscillatory steady state of y when the system was overstimulated with low and high hormone. In the experiment $\mathrm{D}$ there were some small oscillations after the initial spike before reaching the steady nonoscillatory state compared to experiment $\mathrm{C}$.

Properties: The behavior of Hormone-induced Calcium Oscillations in Liver Cells is extracted based on the value of parameters a and a. The main behaviors of the system can be concluded as follows: (1) The system unstable when it is under-stimulated (high value of a) in which the system obtain sustained oscillations and the calcium concentration in cytosol returns back to the base value after each spike of the oscillation. This means that, when the system reaches high number of spikes, there are high hormone channeling. The number of spikes increase with the increase of hormone level (a); (2) The system is stable when it is over-stimulated (low value of a) in which the system achieve a nonoscillatory state after an initial spike regardless of the level of the hormone. Therefore, the properties of the system are formulated from these two behaviors as follow: (1) For experiment $\mathrm{A}$ and $\mathrm{B}$, the reaction of hormone channeling will be actively activated during the formation of spikes with the high concentration of calcium in the cytosol; (2) For experiment C and D, 
hormone channeling is stabilized to achieve a steady non-oscillatory state with low concentration of calcium in the cytosol after a small initial spike.

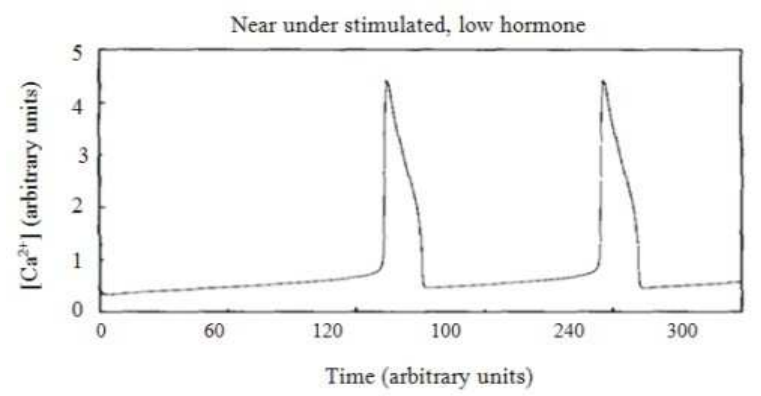

(A)

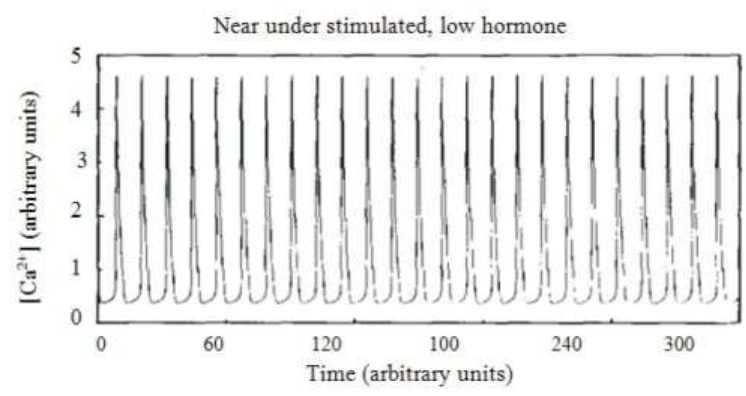

(B)

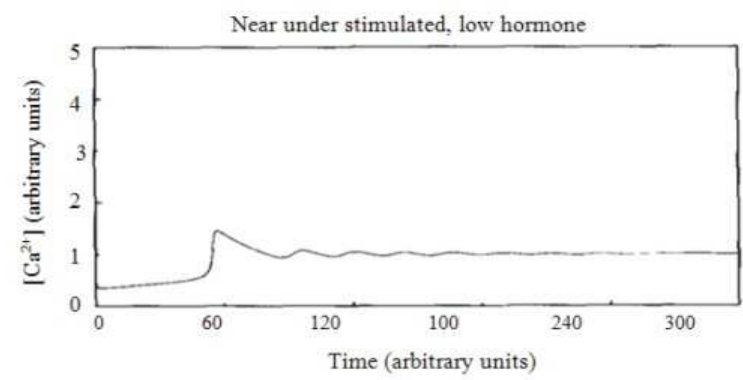

(C)

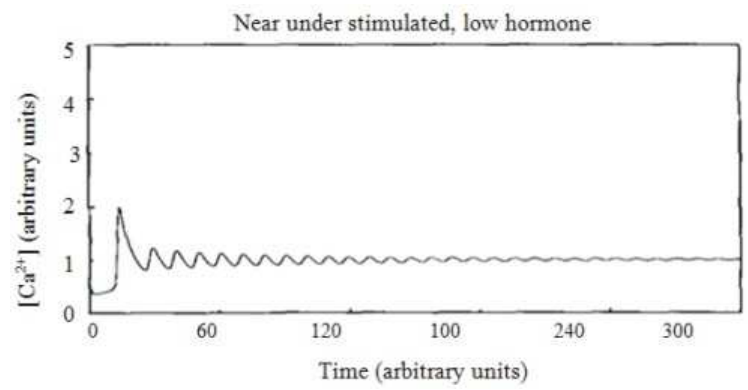

(D)

Fig. 1: ODE simulation of calcium oscillation (Somogyi and Stucki, 1991)
Modeling: The chemical substances, the structure of the system, the processes that interact between compartments and the parameters assigned to each processes are extracted from ODE model (Somogyi and Stucki, 1991). This information is used in modeling the case study with membrane computing formalism as investigated by Muniyandi and Abdullah (2009).

Simulation: Gillespie Simulator is used to simulate the membrane computing model. The parameters are extracted from the mathematical model build by Somogyi and Stucki (1991). The same parameters and initial multisets that were used in ODE model are also used in the membrane computing model. The arbitrary value of $\mathrm{a}$ and $\mathrm{a}$ are chosen to determine the understimulated or overstimulated and the low or high level of the hormone. The selected values for the four different experiments are: (A) $\mathrm{a}=20$ and $\mathrm{a}=5$; (B) $\mathrm{a}=$ 20 and $\mathrm{a}=200(\mathrm{C}) \mathrm{a}=0.2$ and $\mathrm{a}=5 ;(\mathrm{D}) \mathrm{a}=0.2$ and $\mathrm{a}=$ 200. Firstly, the membrane computing model is converted into System Biology Markup Language (SBML) notations which describe the components of the biological system. Then, the simulator will specify the list of compartments and the structural hierarchy and the initial amounts of calcium from the SBML notations. This is the initial state of the system and is given to the simulator to produce an evolution of the calcium in cytosol over simulation steps. The membrane computing simulation results using Gillespie Algorithm are compared to the results of ODE approach.

PRISM model checker: PRISM is probabilistic model checker that represent a technique for formally verifying quantitative properties of a stochastic system. A model to be analyzed is specified in PRISM language, which is a simple, high level, state-based language based on the Reactive Modules formalism (Alur and Henzinger, 1999). PRISM supports a probabilistic model based on continuous time Markov chains and systems specifications through continuous stochastic logic for stochastic systems.

Methods: The biological case study modeled in membrane computing is translated into PRISM formalism. This translation technique from membrane computing into PRISM applied in this research is proposed by Romero-Campero et al. (2006). Then the model in PRISM is simulated and model checked with the properties of the case study. PRISM is used to specify and to analyze properties based on rewards. Rewards are used to reason the behavior of the model in a certain fashion by measuring the probability as well as to identify a wide range of quantitative 
measures relating to modeling behavior. The data of the results generated by PRISM is exported into Scilab (Campbell et al., 2005) to be presented into a graph. Finally, the graph is analyzed to verify whether the identified properties are preserved or not.

\section{RESULTS}

The model of membrane computing: Hormoneinduced calcium oscillations in liver cells is three compartments system with an object (chemical substance) and 5 rules. The membrane system of Hormone-induced Calcium Oscillations in Liver Cells (HCOLC) can be represented as:

$$
\operatorname{HCOLC}=\left(V, \mu, \omega_{E}, \omega_{C}, \omega_{E R}, R_{E}, R_{C}, R_{E R}\right)
$$

The system contains three compartments, that are Extracellular (E), Cytosol (C) and Endoplasmic Reticulum (ER). In this system $\mathrm{C}$ is in the middle interacting with $\mathrm{E}$ and $\mathrm{ER}$. This means that there is no direct linkage between $\mathrm{E}$ and ER. Therefore the membrane structure of this biological system can be represented as:

$$
\left.\mu=\left[[]_{\mathrm{ER}}\right]_{\mathrm{C}}\right]_{\mathrm{E}}
$$

The object in this system is calcium. In order to differentiate the concentration of calcium in each compartments, it is denoted with the symbol of the compartment.

$$
\mathrm{V}=\left\{\mathrm{Ca}_{\mathrm{E}}, \mathrm{Ca}_{\mathrm{C}}, \mathrm{Ca}_{\mathrm{ER}}\right\}
$$

The initial multisets are:

$$
\omega_{\mathrm{E}}=\left\{\mathrm{Ca}_{\mathrm{E}}\right\}, \omega_{\mathrm{C}}=\left\{\mathrm{Ca}_{\mathrm{C}}\right\} \text { and } \omega_{\mathrm{ER}}=\left\{\mathrm{Ca}_{\mathrm{ER}}\right\}
$$

The evolution rule has the form: $R_{i}: u[v]_{i} \stackrel{k}{\longrightarrow} u^{\prime}\left[v^{\prime}\right]_{i}$, where $u, v, u^{\prime}, v^{\prime}$ are multisets and $\mathrm{i}$ is compartment. $\mathrm{k}$ is a real number representing the kinetic constant. The rules in HCOLC system are communication rules. The rules are:

Calcium flux from extracellular into the cytosol:

$$
\mathrm{R}_{\mathrm{C}} 1: \mathrm{Ca}_{\mathrm{E}}[]_{\mathrm{C}} \stackrel{\mathrm{k}_{1}}{\longrightarrow}\left[\mathrm{Ca}_{\mathrm{C}}\right]_{\mathrm{C}}, \mathrm{k}_{1}=\gamma
$$

Calcium translocation from cytosol to ER:

$$
\mathrm{R}_{\mathrm{ER}} 1: \mathrm{Ca}_{\mathrm{C}}[]_{\mathrm{ER}} \stackrel{\mathrm{k}_{2}}{\longrightarrow}\left[\mathrm{Ca}_{\mathrm{ER}}\right]_{\mathrm{ER}}, \mathrm{k}_{2}=\mathrm{k}^{\prime} * \mathrm{Ca}_{\mathrm{C}}
$$

Calcium translocation from ER to cytosol:

$$
\mathrm{R}_{\mathrm{ER}} 2:\left[\mathrm{Ca}_{\mathrm{ER}}\right]_{\mathrm{ER}} \stackrel{\mathrm{k}_{3}}{\longrightarrow} \mathrm{Ca}_{\mathrm{C}}[]_{\mathrm{ER}}, \mathrm{k}_{3}=\mathrm{k} * \mathrm{Ca}_{\mathrm{ER}}
$$

Calcium pumped out of cytosol into extracellular:

$$
\begin{gathered}
\mathrm{R}_{\mathrm{C}} 2:\left[\mathrm{Ca}_{\mathrm{C}}\right]_{\mathrm{C}} \stackrel{\mathrm{k}_{4}}{\longrightarrow} \mathrm{Ca}_{\mathrm{E}}[]_{c}, \mathrm{k}_{3}=\mathrm{k} * \mathrm{Ca}_{\mathrm{ER}} \\
\mathrm{R}_{\mathrm{ER}} 3:\left[\mathrm{Ca}_{\mathrm{ER}}\right]_{\mathrm{ER}} \stackrel{\mathrm{k}_{\mathrm{s}}}{\longrightarrow} \mathrm{Ca}_{\mathrm{C}}[]_{\mathrm{ER}}, \mathrm{k}_{5}=\alpha * \mathrm{f}\left[\mathrm{Ca}_{\mathrm{C}}\right] * \mathrm{Ca}_{\mathrm{ER}}
\end{gathered}
$$

Simulation: Membrane computing model of HCOLC is evaluated by using Gillespie algorithm by using multicompartment Gillespie simulator as used by Muniyandi and Abdullah (2010). This experiment intended to analyze how parameters a and a affect the oscillatory and nonoscillatory behavior of Calcium in cytosol of the HCOLC system. This behavior is to determine the stability of the system. Figure 2 shows the results generated by these experiments. $2 \mathrm{~A}$ and $\mathrm{B}$ shows that the processes in the system occur with the understimulated environment when the value of a is high. If the hormone level low as shown in $2 \mathrm{~A}$ then there are less spikes compared to when the hormone level is high as in $2 \mathrm{~B}$. Meanwhile, the processes in the system are overstimulated when the value of $\alpha$ is low but the low or high hormone level do not affect the behavior of the system as shown in $2 \mathrm{C}$ and $\mathrm{D}$.

Model checking with PRISM: The membrane computing model translated into PRISM language as below:

ctmc

// initial amount of calcium const int $\mathrm{X} \_$bound $=\mathrm{N}$; //X is calcium in ER const int $\mathrm{Y} \_$bound $=\mathrm{N} ; / / \mathrm{Y}$ is calcium in cytosol const int $\mathrm{N}$; / maximum amount of calcium in the system

// base rates of parameters

const double $\mathrm{k}=0.01$;

const double alpha $=5.0$;

const double $\mathrm{k} 1=2.0$;

const double beta $=1.0$;

const double gamma $=1.0$;

const double $\mathrm{a}=20.0$;

module HCOLC

$\mathrm{X}:[0 . . \mathrm{X}$ _bound $]$ init 1 ;

Y : [0..Y_bound] init 1;

[R1] $\mathrm{Y}<\mathrm{Y}$ _bound -> gamma: $\left(\mathrm{Y}^{\prime}=\mathrm{Y}+1\right)$;

[R2] $X>0$ and $Y<Y$ bound $->k^{*} X:\left(X^{\prime}=X-1\right)$ and

$\left(\mathrm{Y}^{\prime}=\mathrm{Y}+1\right)$;

[R3] $\mathrm{Y}>0$ and $\mathrm{X}<\mathrm{X} \_$bound $->\mathrm{k} 1 * \mathrm{Y}:\left(\mathrm{Y}^{\prime}=\mathrm{Y}-1\right)$ and

$\left(X^{\prime}=\mathrm{X}+1\right)$;

[R4] Y>0 -> beta*Y : $\left(\mathrm{Y}^{\prime}=\mathrm{Y}-1\right)$;

[R5] X>0 \& $Y<Y \_$bound ->

alpha*X*(pow $(\mathrm{Y}, 4) /(\operatorname{pow}(\mathrm{a}, 4)+\operatorname{pow}(\mathrm{Y}, 4)))$ :

$\left(\mathrm{X}^{\prime}=\mathrm{X}-1\right)$ and $\left(\mathrm{Y}^{\prime}=\mathrm{Y}+1\right)$; endmodule 


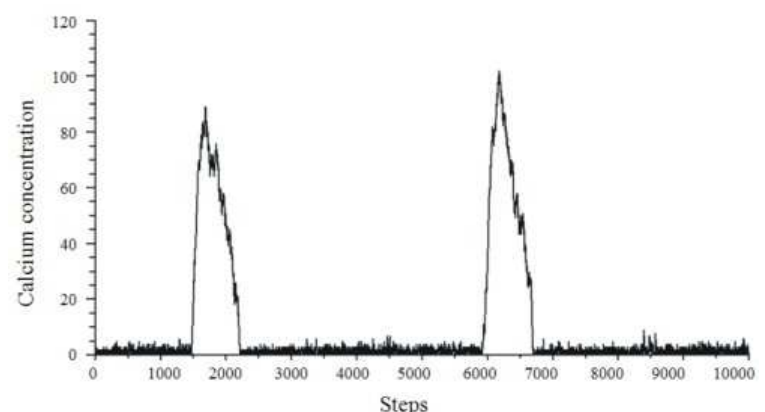

(A)

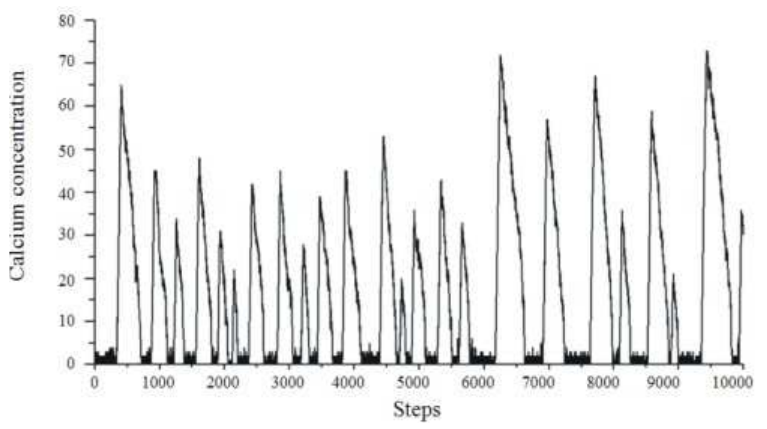

(B)

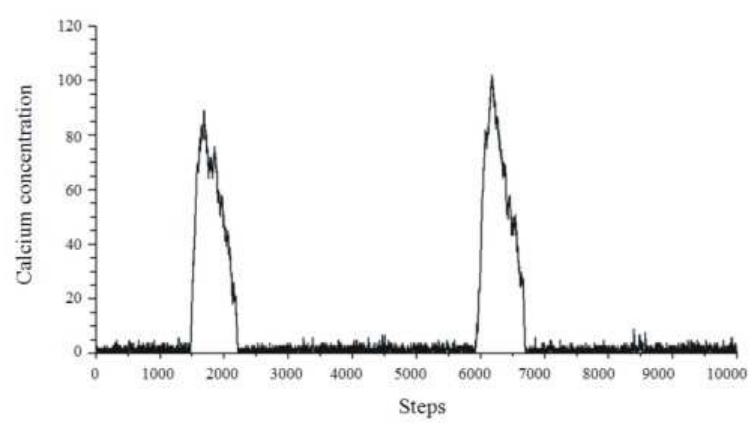

(C)

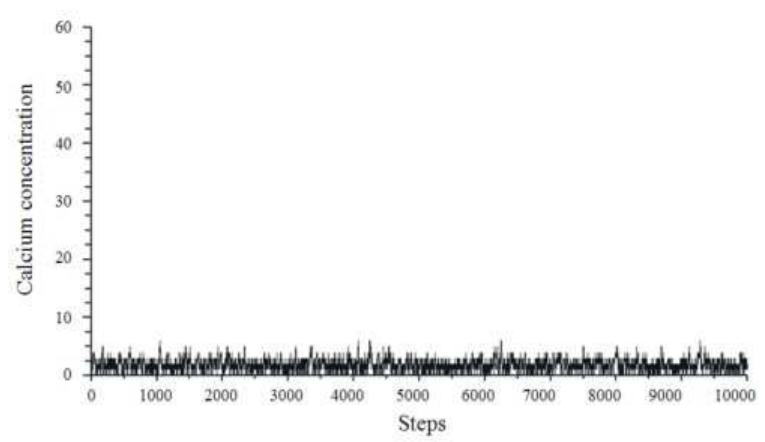

(D)

Fig. 2: Experiments with the value of a and a (A) $\mathrm{A}=$ 20 and $\mathrm{A}=5$ (B) Experiment $\mathrm{B}: \mathrm{a}=20$ and $\mathrm{a}=$ 200 (C) Experiment $\mathrm{C}: \mathrm{a}=0.2$ and $\mathrm{a}=200$ (D) Experiment D: $\mathrm{a}=0.2$ and $\mathrm{a}=200$
By using the concept of rewards, PRISM is used to specify and to analyze the two properties of HCOLC system as described above. The rewards "Flux_In", "translocation_cytosol", "translocation_ER", "Flux_Out" and "HormoneChanneling" are to verify the activation of processes (R1, R2, R3, R4 and R5) in the system for each experiments (A, B, C and D). For each rewards, when the process is activated, it will determine the concentration of calcium in cytosol. For instance for the rewards "Flux_In", the calcium (Y) concentration is indicated when the process R1 is activated during the simulation. The rewards are described as follow:

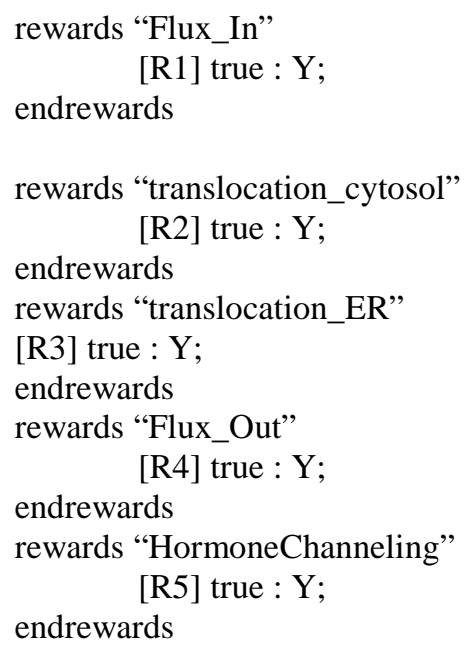

Property (1): This property is analyzed with experiment $\mathrm{A}$ and $\mathrm{B}$ in Fig. 3 and 4. The model checking results of these two experiments show that the hormone channeling is high when the spikes are formed as shown by R5 in Fig. 3 and 4. This process is stabilized by regular occurrence of the flux-out process (R4) and translocation process from cytosol to ER (R3). Meanwhile the flux-in Process (R1) and translocation process from ER to cysotol (R2) are suppressed. More regular spikes are occurring when the hormone level is high with increasing hormone channeling (R5) as shown by Fig. 4 with experiment B.

Property (2): This property is analyzed with experiment $\mathrm{C}$ and $\mathrm{D}$. Both experiments generate approximately the same results as shown in the Fig. 5. The model checking results of these two experiments show that the hormone channeling (R5) is moderate and the spikes are not formed at the start of the simulation but in the ODE model there was a spike at initial stage of the simulation. This process is stabilized by regular occurrence of the flux-out process (R4) and translocation process from cytosol to ER (R3). Meanwhile the flux-in process (R1) and translocation process from ER to cysotol (R2) are suppressed. 


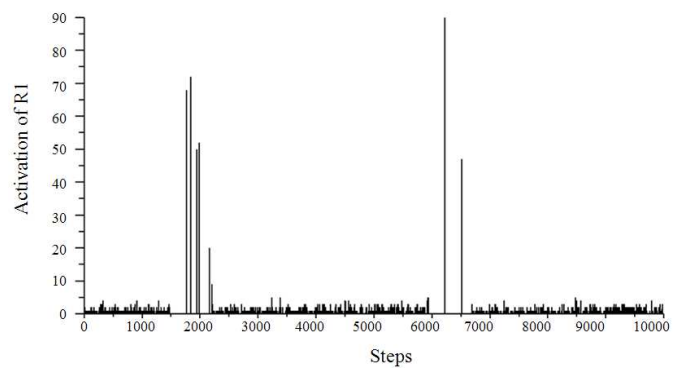

(a)

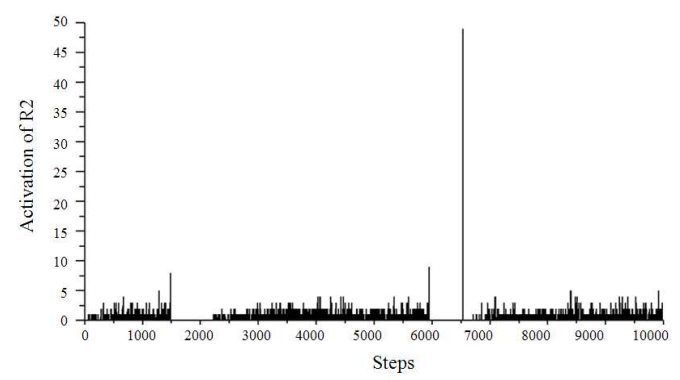

(b)

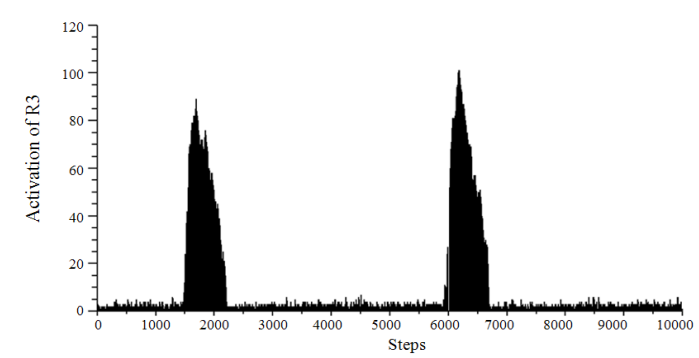

(c)

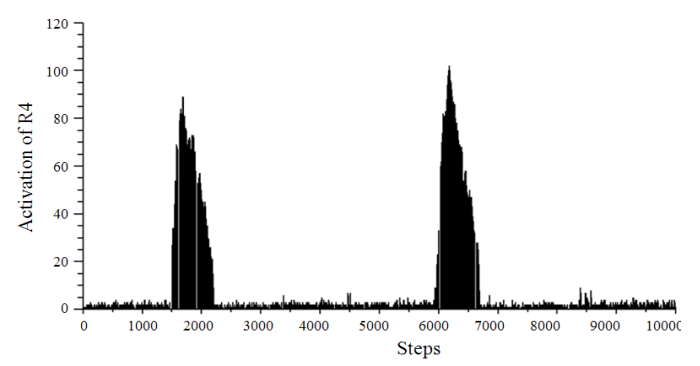

(d)

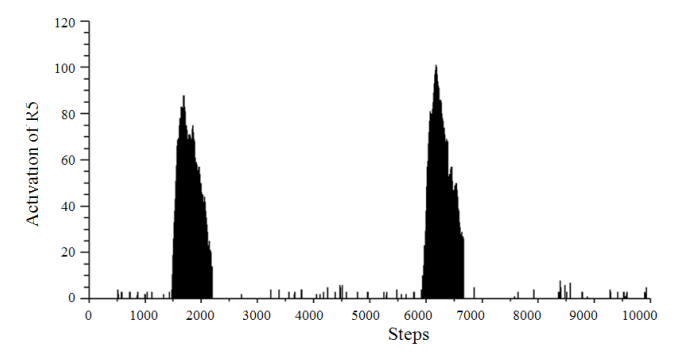

(e)

Fig. 3: Activation of the processes in the experiment A. (a) R1; (b) R2; (c) R3; (d) R4; (e) R5

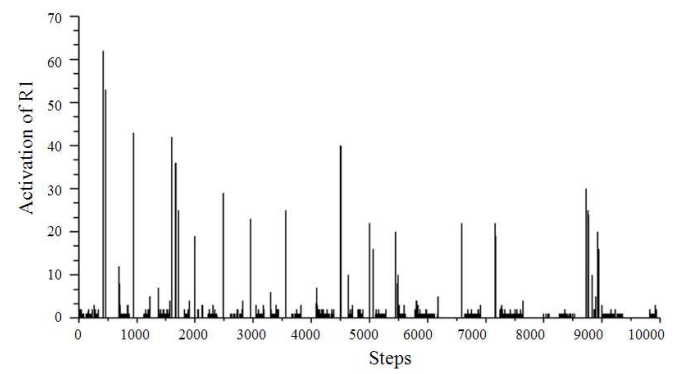

(a)

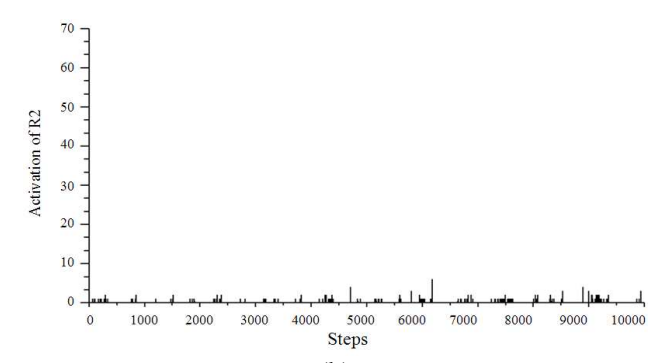

(b)

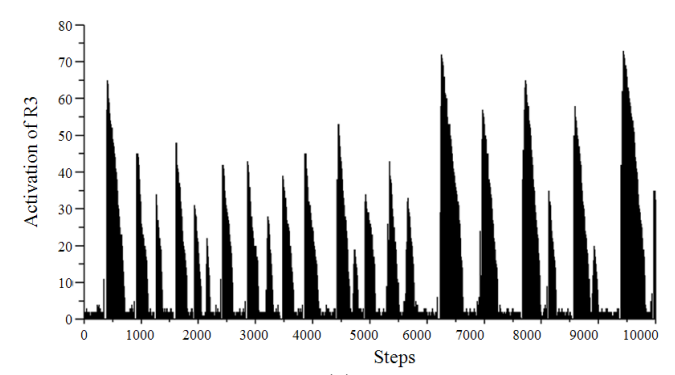

(c)

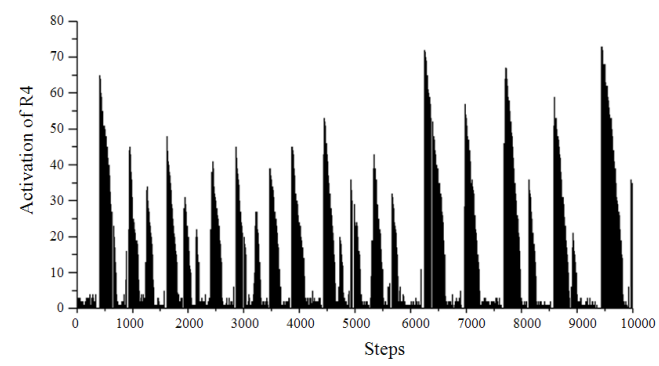

(d)

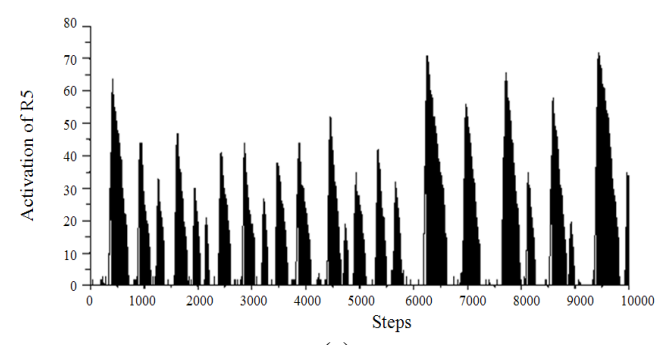

(e)

Fig. 4: Activation of the processes in the experiment B. (a) R1; (b) R2; (c) R3; (d) R4; (e) R5 


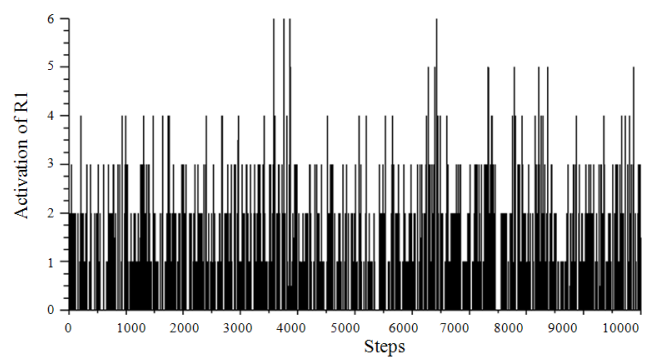

(a)

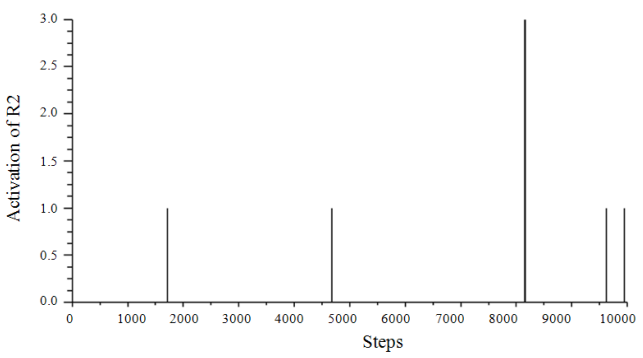

(b)

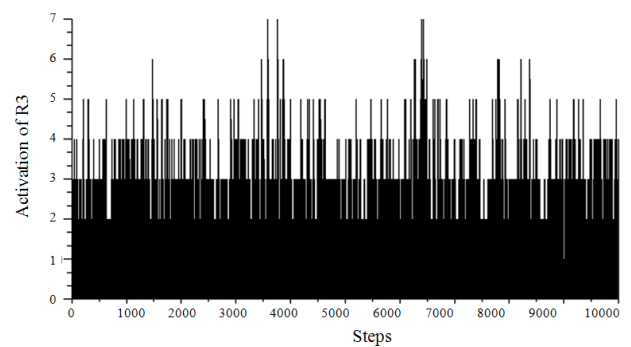

(c)

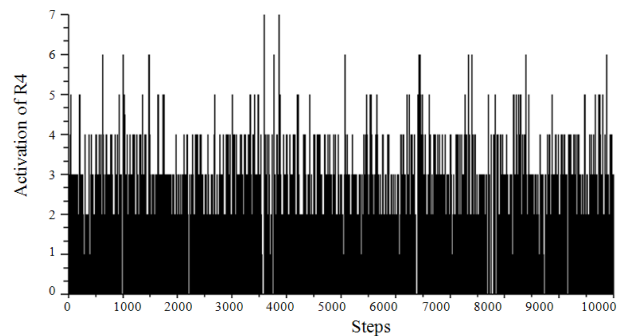

(d)

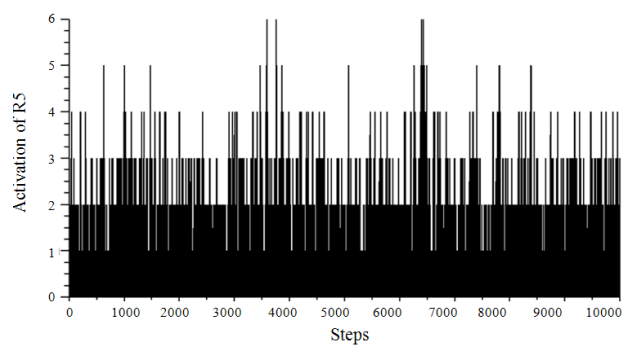

(e)

Fig. 5: Activation of the processes with the experiment C and D. (a) R1; (b) R2; (c) R3; (d) R4; (e) R5

\section{DISCUSSION}

The modeling of the HCOLC system shows that modeling of biological system with membrane computing provides better option to symbolize the structure and the processes involve in biological system compared to ODE approach. This is because we able to distinguish the processes and the movement of objects between compartments in this hierarchical system. Meanwhile, the ODE model emphasizes on the processes of the overall system but has ignored the structure of the system and the character of processes involve in each of the compartments.

The simulation results show that membrane computing model provides approximately similar results that were generated in the ODE model. However, with membrane computing model, the non-deterministic and parallel executions of processes in different compartments of biological system are obvious compared to the deterministic approach of ODE.

However, the slight differences between the experiment $\mathrm{C}$ and $\mathrm{D}$ could not be differentiated with the simulation of membrane computing model. With the ODE model as in the experiment C and D as in Fig. 1, it showed that after an initial spike, calcium adopts steady state value following more or less small frequency of oscillations and it will be more pronounced with the high hormone as in the experiment D. In the membrane computing model, the initial spike is not obvious though there is steady state of small frequency of oscillations. This is because the membrane computing model works discreetly and stochastically with the same value of parameters that provided in the continuous model of ODE. Therefore membrane computing model could not differentiate the small changes between the two experiments as shown by the ODE model.

Nonetheless, the basic properties of the system have been preserved in the model as shown by the model checking results. These results demonstrate how different processes in each of the compartments play role in contributing to the behavior of the overall system.

\section{CONCLUSION}

The investigation demonstrates that the membrane computing model not only capable to capture the structure and the processes involve in the multicompartments biological system but also able to preserve the behavior and the properties of the system. Therefore, membrane computing is capable of representing multi-compartment biological system in a formal way without disregarding its biological characteristics. This means that the membrane 
computing able to address the limitation of the ODE model in representing biological system.

However there are some of membrane computing features that could still be extended to strengthen its capability in modeling biological system. One such issue is the selection of kinetic constants for stochastic system of membrane computing. The result of experiment $\mathrm{C}$ and $\mathrm{D}$ shows that the appropriate parameters or kinetic constants to prioritize the stochastic selection of the rules to determine the execution of specific process at certain time steps have to correctly selected based on the behavior of the system. The attempt in this investigation to use the same value of parameters as in the ODE model to implement membrane computing model could not generate the intended results as in the experiment $\mathrm{C}$ and D. Therefore, the parameters chosen should be based on the stochastic characteristics of the system. The method to address this issue is under investigation.

\section{REFERENCES}

Alur, R. and T.A. Henzinger, 1999. Reactive modules. Formal Methods Syst. Des., 15: 7-48. DOI: 10.1023/A:1008739929481

Bernardini, F. and M. Gheorghe, 2004. Population P systems. J. Univ. Comput. Sci., 10: 509-539. DOI: 10.3217/jucs-010-05-0509

Bernardini, F.M., N. Gheorghe, R.C. Krasnogor, M.J. Muniyandi and Perez-Jimenez et al., 2006. On $\mathrm{P}$ systems as a modeling tool for biological systems. Lecture Notes Comput. Sci., 3850: 114-133. DOI: 10.1.1.144.5278

Campbell, S.L., J.P. Chancelier and R. Nikoukhah, 2005. Modeling and Simulation in Scilab/Scicos1st Edn., Springer, USA., ISBN: 10: 0-387-278028, pp: 313.
Gillespie, D.T., 2001. Approximate accelerated stochastic simulation of chemically reacting systems. J. Chem. Phys., 115: 1716-1733.

Kwiatkowska, M., G. Norman and D. Parker, 2002. PRISM: Probabilistic symbolic model checker. Lecturer Notes Comput. Sci., 2324: 113-140. DOI: 10.1007/3-540-46029-2_13

Muniyandi, R. and M.Z. Abdullah, 2009. Modeling of biological processes by using membrane computing formalism. Am. J. Applied Sci., 6: 1961-1969. DOI: 10.3844/ajassp.2009.1960.1968

Muniyandi, R. and M.Z. Abdullah, 2010. Experimenting the simulation strategy of membrane computing with Gillespie algorithm by using two biological case studies. J. Comput. Sci., 6: 525-535.

Muniyandi, R., M.Z. Abdullah and Z. Shukor, 2010. Model checking the biological model of membrane computing with probabilistic symbolic model checker by using two biological systems. J. Comput. Sci., 6: 669-678.

Paun, G., 1998. Computing with membranes. J. Comput. Syst. Sci., 61: 108-143. DOI: 10.1006/jcss.1999.1693

Romero-Campero, F.S, M. Gheorghe, L. Bianco, D. Pescini and M.J. Perez-Jimenez et al., 2006. Towards probabilistic model checking on $\mathrm{P}$ systems using PRISM. Lecture Notes Comput. Sci., 4361: 477-495. DOI: 10.1007/11963516_30

Somogyi, R. and J.W. Stucki, 1991. Hormone-induced calcium oscillations in liver cells can be explained by a simple one pool model. J. Biol. Chem., 266: 11068-11077. http://www.jbc.org/content/266/17/11068.full.pdf 